\title{
Channel-aware adaptive receivers for linearly precoded MIMO-OFDM systems with imperfect CSIT
}

\author{
Felip Riera-Palou* and Guillem Femenias
}

\begin{abstract}
Within the context of linearly precoded MIMO-OFDM (combination of multiple antenna techniques with multicarrier transmission schemes such as orthogonal frequency division multiplexing) systems with multiple-stream transmission, maximum likelihood detection (MLD) has been shown to offer large performance gains when compared to an all-linear setup (i.e., linear transmitter/receiver) when either perfect or imperfect channel state information at the transmitter (CSIT) is available. Unfortunately, these gains come at the cost of a higher complexity. In particular, the increase in computational cost is more significant when the receiver is designed to operate with soft information and even more dramatic when, in order to optimise error rate performance, iterative decoding is allowed. In order to exploit the best features of each detection technique, this paper proposes a method to selectively choose the detection strategy (ML or linear) for each individual subcarrier as a function of the instantaneous channel conditions and CSIT accuracy. Numerical results show that a cautious and selective use of ML detection substantially reduces complexity while still reaping most of the performance advantage.
\end{abstract}

\section{Introduction}

The combination of multiple antenna techniques (socalled MIMO) with multicarrier transmission schemes such as orthogonal frequency division multiplexing (OFDM), the so-called MIMO-OFDM architecture, is now at the heart of most state-of-the-art wireless systems and future standards [1-3]. In this context, techniques that exploit the availability of channel state information at the transmitter (CSIT) have been intensively researched (see $[4,5]$ for a review). It is well known that the capacityachieving strategy when perfect CSIT is available is to pre-cancel interference among simultaneously transmitted streams, a scheme usually referred to as dirty paper coding (DPC) [6]; however, its high computational cost motivates the need for simpler strategies. Palomar et al., in their landmark paper [7], introduced a framework for the optimisation of MIMO-OFDM systems with CSIT based on linear processing at the transmitter and receiver. The proposed scheme defines transmit and receiver filters that are based on the singular value decomposition (SVD) of

\footnotetext{
*Correspondence: felip.riera@uib.cat

Mobile Communications Group, Department of Mathematics and Informatics, University of the Balearic Islands, Mallorca, Illes Balears 07122, Spain
}

the whitened channel matrix and performs a distribution of the available power among the different transmit modes using waterfilling in accordance with various performance metrics. Further insight on this architecture was provided in $[8,9]$, where the diversity order performance was analysed for single- and multiple-stream configurations (i.e. spatial division multiplexing beamforming). These studies showed that such schemes lose diversity when increasing the number of transmitted streams as performance becomes dominated by the worst employed spatial transmission mode. Very recently, it has been shown in [10] that full diversity can be restored by incorporating a linear transformation at transmission spreading the symbols to be transmitted over the available spatial modes. Unfortunately, this diversity advantage comes at the cost of having to rely on joint maximum likelihood detection (MLD) at the receiver.

Most of these results assumed perfect CSIT, which is a rather optimistic hypothesis in practical deployments. Channel feedback delay and quantization noise are typical impairments affecting the quality of CSIT, whose effects should be accounted for. To this end, [11] incorporated channel knowledge imperfections in the design

\section{Springer}

(c) 2013 Riera-Palou and Femenias; licensee Springer. This is an Open Access article distributed under the terms of the Creative Commons Attribution License (http://creativecommons.org/licenses/by/2.0), which permits unrestricted use, distribution, and reproduction in any medium, provided the original work is properly cited. 
of a linear transmitter/receiver architecture that, as the CSIT approaches perfection, converges towards the solution of [7]. A related work by Sengul et al. [12] proposes a codebook construction methodology based on a Lloyd quantizer design that aims at the improvement of the robustness against imperfect CSIT in linearly precoded bit-interleaved coded modulation (BICM) systems while still relying on linear filters at the receiver side. In [13], precoding strategies combined with forward error correction were considered but again limiting the context to that of linear detectors. Remarkably, it should be noted that, under imperfect CSIT, the optimisation of error rate metrics requires MLD-based reception.

The use of MLD in combination with linear precoding has been extensively studied in [14] and [15] under perfect and imperfect CSIT, showing that large reductions in the bit/packet error rate (BER/PER) are possible at the cost of an increased receiver complexity as even smart implementations (i.e. sphere decoding [16]) are computationally demanding at low signal-to-noise ratios (SNRs), where practical systems usually operate [17]. To address this downside, this paper proposes the selective and careful application of MLD only under very specific conditions, which depend on the specific channel realisation and CSIT accuracy, while otherwise relying on linear detection. The introduced technique is shown to be effective with various architectures, namely hard-, softand iteratively decoded receivers. This scheme is specially appropriate for scenarios where the channel and/or CSIT accuracy may vary widely from packet to packet. As an illustrative example of this type of scenario, this paper considers wireless local area networks (WLANs) based on the IEEE 802.11n standard [18], whose multipleaccess policy based on carrier sense multiple access with collision avoidance (CSMA/CA) causes substantial variations in the accuracy of the available channel information at the transmitter. It is worth mentioning that a related idea, but in the context of MIMO systems without CSIT and restricted to the $2 \times 2$ MIMO setup, was introduced in [19], where the detection strategy selection was based on the condition number of the channel correlation matrix resulting in the utilisation of linear and ML detection for well-conditioned and ill-conditioned channels, respectively.

The rest of the paper is organised as follows: Section 2 introduces the system model under consideration including a description of the assumptions regarding the channel model and CSIT accuracy. Section 3 begins by reviewing the two classic detectors at hand, minimum mean square error (MMSE) and MLD, within the context of the considered scenario and subsequently introduces the channel-aware adaptive detector. In Section 4, the adaptive detector concept is revisited within the framework of soft and iterative detection strategies. Numerical results are presented in Section 5 illustrating the benefits of the channel-aware adaptive detector. Finally, the main outcomes of this work are recapped in Section 6.

This introduction concludes with a brief notational remark. Vectors and matrices are denoted by bold lower case and bold upper case letters, respectively. The superscript $(\cdot)^{H}$ denotes the complex transpose (Hermitian) of the corresponding variable. The symbol $\boldsymbol{I}_{k}$ denotes the $k$-dimensional identity matrix, whereas $\mathcal{D}(\boldsymbol{x})$ is used to represent a (block) diagonal matrix having $x$ at its main (block) diagonal and $[\boldsymbol{A}]_{i, j}$ serves to indicate the $(i, j)$-element of matrix $A$.

\section{System model}

A MIMO-OFDM architecture is considered where the transmitter and receiver are equipped with $N_{T}$ and $N_{R}$ antennas, respectively, which are capable of simultaneously transmitting $N_{s} \leq \min \left(N_{T}, N_{R}\right)$ data streams. The available system bandwidth is exploited by means of $N_{c}$ subcarriers out of which $N_{d}$ are used to carry data and $N_{p}$ are destined to pilot signals and guard bands.

\subsection{Transmitter processing}

Following the usual processing steps of BICM systems, incoming information packets are first channel encoded and possibly punctured to satisfy prescribed rate constraints, and the resulting bits are then distributed among $N_{s}$ streams corresponding to different spatial branches. On each spatial branch, bits are interleaved and mapped onto modulation symbols drawn from an $M$ ary modulation alphabet, resulting in the set of symbol streams $\left\{\mathbf{s}_{1}, \cdots, \mathbf{s}_{N_{s}}\right\}$. Each spatial stream is then organised into segments of $N_{d}$ symbols that will eventually become OFDM symbols (with the addition of pilot/null subcarriers) (Figure 1).

The mapping from information to transmit symbols (i.e. precoding) on subcarrier $q$ at discrete time instant $n$ is carried out as

$$
\mathbf{x}[q, n]=\mathbf{W}[q, n] \mathbf{s}[q, n]
$$

where $\mathbf{W}[q, n]$, with dimensions $N_{T} \times N_{s}$, represents the precoding matrix and $\mathbf{s}[q, n]=\left(s_{1}[q, n] \cdots s_{N_{s}}[q, n]\right)^{T}$, with $s_{i}[q, n]$ denoting the symbol corresponding to the $i$ th stream to be transmitted on the $q$ th subcarrier at time instant $n$. Finally, the precoded symbols are supplied to an OFDM modulator consisting of an IFFT plus the addition of a cyclic prefix (CP).

\subsection{Channel modelling}

The channel between an arbitrary pair of $\mathrm{Tx}$ and $\mathrm{Rx}$ antennas is assumed to be frequency-selective with a scenario-dependent power delay profile common to all Tx-Rx pairs. Let us denote by $\mathbf{H}[q, n]$ the matrix representing the channel frequency response on the $q$ th subcarrier 


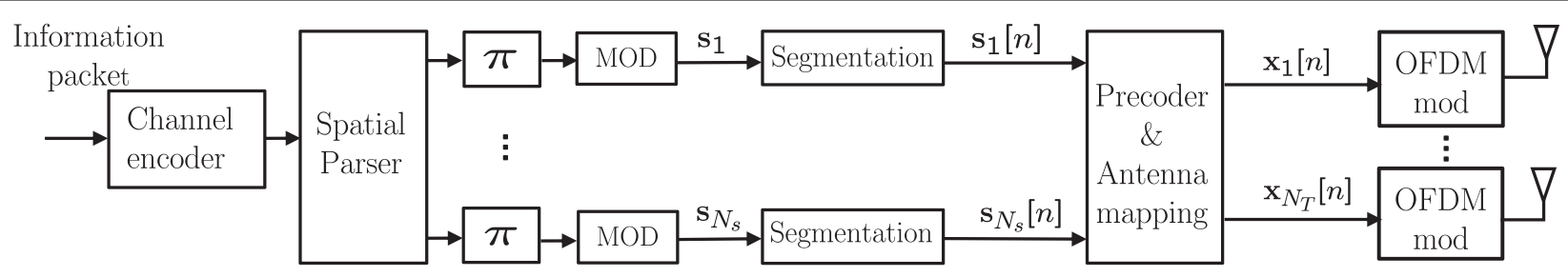

Figure 1 Transmitter block diagram for linearly precoded system.

$$
\mathbf{H}[q, n]=\left(\begin{array}{ccc}
h_{1,1}[q, n] & \cdots & h_{1, N_{T}}[q, n] \\
\vdots & & \vdots \\
h_{N_{R}, 1}[q, n] & \cdots & h^{N_{R}, N_{T}}[q, n]
\end{array}\right),
$$

where an arbitrary entry $h_{i, j}[q, n]\left(1 \leq i \leq N_{R}, 1 \leq j \leq\right.$ $N_{T}$ ) corresponds to the frequency response on subcarrier $q$ of the channel linking Tx antenna $j$ and Rx antenna $i$. It is assumed that the entries of $\mathbf{H}[q, n]$ are uncorrelated ${ }^{\mathrm{a}}$ (i.e. $\mathrm{Tx} / \mathrm{Rx}$ antennas are sufficiently spaced).

Channel estimation at the receiver is considered accurate enough so as to render any CSI error at the Rx side (CSIR) negligible. In contrast, CSIT is considered to be imperfect in such a way that [11]

$$
\mathbf{H}[q, n]=\rho[n] \overline{\mathbf{H}}[q, n]+\sqrt{1-\rho[n]^{2}} \boldsymbol{\Delta}[q, n],
$$

where $\overline{\mathbf{H}}[q, n]$ represents the channel mean known at the transmitter (estimated channel), $\Delta[q, n]$ denotes the channel estimation noise whose entries are $\mathcal{C N}(0,1)$, and $\rho[n] \in[0,1]$ can be a packet-dependent random variable effectively modelling the CSIT accuracy for the current packet, which is also known at the receiver side.

\subsection{Reception equation}

Figure 2 depicts a generic receiver based on hard decoding. Reception begins with the standard OFDM demodulation consisting of the $\mathrm{CP}$ removal and FFT processing. Perfect $\mathrm{Tx} / \mathrm{Rx}$ synchronisation and a CP length exceeding the duration of the channel impulse response are assumed, thus guaranteeing that consecutive OFDM symbols do not suffer from inter-block interference. Under these conditions, the detection procedure works on an OFDM symbol basis, thus allowing us to drop the timerelated index $n$ from subsequent equations (e.g. $\mathbf{s}[q, n] \rightarrow$ $\mathbf{s}[q]$ ). The received baseband samples on subcarrier $q$, denoted by $\mathbf{r}[q]=\left[r_{1}[q] \cdots r_{N_{r}}[q]\right]^{T}$ with $r_{i}[q]$ representing the received sample on the $i$ th receive antenna, for an arbitrary OFDM symbol are given by

$$
\mathbf{r}[q]=\mathbf{A}[q] \mathbf{s}[q]+\boldsymbol{v}[q],
$$

where $\mathbf{A}[q]=\mathbf{H}[q] \mathbf{W}[q]$, and the $N_{R} \times 1$ vector $\boldsymbol{v}[q]$ corresponds to the noise samples affecting the $q$ th subcarrier, which are assumed to be i.i.d. and drawn from a zero-mean complex Gaussian distribution with variance $\sigma_{v}^{2}$. It is assumed that, on average, each subcarrier has unit energy available to transmit $N_{s}$ symbols and that the channel frequency response is normalised so that the average signal-to-noise ratio per subcarrier can be defined as $E_{s} / N_{0}=1 /\left(N_{s} \sigma^{2}\right)$.

\section{Channel-aware robust detection}

In [11] a linear architecture is proposed able to robustly cope with CSIT imperfections. In particular, a practical (uncoded/hard decoded) BER minimisation approach consists of a precoding filter defined by

$$
\mathbf{W}[q]=\mathbf{U}[q] \Omega[q] \mathbf{C},
$$

where $\mathbf{U}[q]$ has as columns the eigenvectors of $\overline{\mathbf{R}}_{q}=$ $\rho^{2} \overline{\mathbf{H}}[q]^{H} \overline{\mathbf{H}}[q]+N_{s} \frac{1}{\sigma_{v}^{2}}$ corresponding to its $N_{s}$ largest eigenvalues, and $\Omega[q]=\mathcal{D}\left(\omega_{q}^{1} \cdots \omega_{q}^{N_{s}}\right)$ is the power allocation matrix whose coefficients can be found optimising a prescribed objective metric [7]. The matrix $\mathbf{C}$ is a (subcarrier-independent) unitary transform that spreads the incoming symbols among the different spatial modes. It has been recently shown in [10] that choosing $\mathbf{C}$ to be the product of a unitary transform (e.g. Fourier, Hadamard) and a constellation rotation, in the form of a

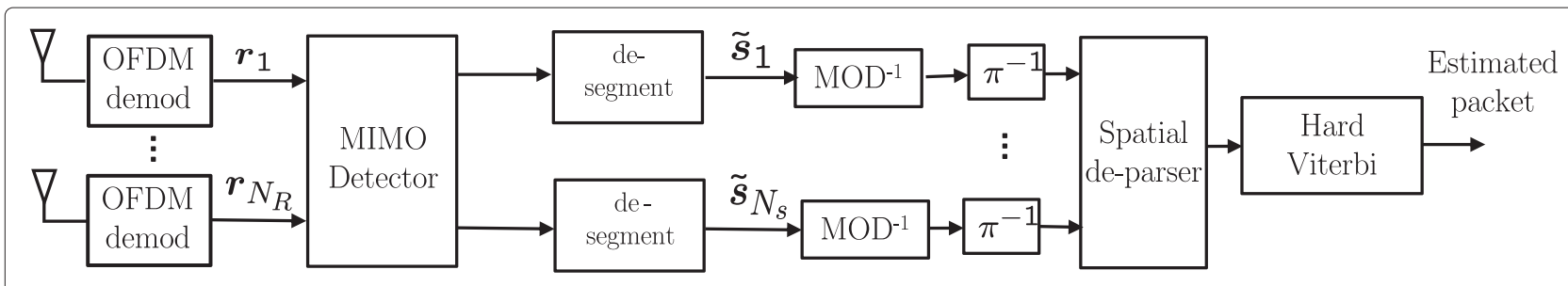

Figure 2 Receiver block diagram with hard detection. 
diagonal matrix with different phase factors, maximises diversity and leads to optimum performance in terms of BER.

\subsection{Linear and non-linear detectors}

Given the received samples in (4), the optimum linear MMSE receive filter is given by [11]

$$
\mathbf{G}[q]=\left(\mathbf{A}[q] \mathbf{A}^{H}[q]+\sigma^{2} \mathbf{I}_{N_{R}}\right)^{-1} \mathbf{A}[q],
$$

allowing (hard) symbol estimates to be obtained as

$$
\tilde{\mathbf{s}}[q]^{\mathrm{MMSE}}=\mathbf{G}^{H}[q] \mathbf{r}[q] .
$$

This detector is also BER optimal when two conditions are met:

1. The CSIT is perfect.

2. The rotation matrix $\mathbf{C}$ in (5) is diagonal.

Under these two conditions, the overall precoderchannnel-detector chain is perfectly diagonalized, thus allowing symbol-by-symbol detection without any performance penalty. Nevertheless, note that condition 2, despite simplifying the detection procedure, inherently induces a diversity loss as it implies that, in loaded setups $\left(N_{s}=\min \left\{N_{T}, N_{R}\right\}\right)$, some of the symbols are transmitted on weak spatial modes that will dominate the error performance [8]. Very recently, [14] and [15] have studied what gains are achieved when one or both of these conditions are not fulfilled and the receiver linearity constraint is neglected, thus allowing the application of MLD. In this case, symbol estimates are given by

$$
\tilde{\mathbf{s}}[q]^{\mathrm{ML}}=\underset{\mathbf{s}[q]}{\operatorname{argmin}}|\mathbf{r}[q]-\mathbf{A}[q] \mathbf{s}[q]|^{2} .
$$

Results in $[14,15]$ show that, in fully loaded configurations, (8) is very advantageous over (7) in terms of BER, although this comes at the cost of an increased receiver complexity, even when employing efficient implementations such as sphere decoding. Regardless of the detection method, either MMSE or ML, estimated symbols are then demodulated, and the corresponding bits, subsequently de-interleaved, (spatially) de-parsed and finally supplied to a Viterbi decoder to obtain an estimate of the transmitted packet.

\subsection{Adaptive detector}

Assuming that the receiver has knowledge of the precoding matrix used by the transmitter ${ }^{\mathrm{b}}$, a detection strategy decision can be made based on the instantaneous channel realisation and specific CSIT accuracy. To this end, let the linear receiver form the overall processing matrix,

$$
\mathbf{G}^{H}[q] \mathbf{A}[q]=\left(\begin{array}{cccc}
\lambda_{1}[q] & \beta_{1,2}[q] & \cdots & \beta_{1, N_{s}}[q] \\
\beta_{2,1}[q] & \lambda_{2}[q] & \cdots & \beta_{2, N_{s}}[q] \\
\vdots & \vdots & \ddots & \vdots \\
\beta_{N_{s}, 1}[q] & \beta_{N_{s}, 2}[q] & \cdots & \lambda_{N_{s}}[q]
\end{array}\right),
$$

where the diagonal terms $\lambda_{1}[q], \cdots, \lambda_{N_{s}}[q]$ correspond to the eigenvalues of $\overline{\mathbf{R}}_{q}$ and form the set of accessible spatial modes available for transmission. There is an intimate connection between the two conditions guaranteeing the optimality of MMSE detection and the structure of (9):

- When conditions 1 and 2 hold, it is obvious that $\beta_{i, j}[q]=0 \forall i, j$ and MMSE detection is optimum.

- When condition 1 holds and condition 2 does not hold, the magnitude of the interfering terms $\beta_{i, j}[q]$ depends on the conditioning of $\mathbf{H}[q]$. If the matrix is well conditioned, MMSE will perform well, but if it is not, MLD will result in a significant advantage.

- When condition 1 does not hold and condition 2 holds, it will depend on the actual realisation of parameter $\rho$. If $\rho \simeq 1$, the overall processing matrix will be virtually diagonalized, making MMSE detection optimal. In contrast, the further away $\rho$ is from 1 , the more significant interfering terms $\beta_{i, j}[q]$ will become, thus requiring MLD for acceptable performance.

- When conditions 1 and 2 do not hold, the detection strategy selection will depend on both the channel matrix conditioning and the specific CSIT accuracy.

To find a decision criterion able to determine the most appropriate detection strategy in light of the instantaneous conditions of the system, let us define $\mathrm{SNR}_{i}[q]$ as

$$
\mathrm{SNR}_{i}[q]=\frac{\left|\lambda_{i}[q]\right|^{2}}{\sigma_{n}^{2}} .
$$

Similarly, the signal-to-interference plus noise ratio (SINR) can be defined as

$$
\operatorname{SINR}_{i}[q]=\frac{\left|\lambda_{i}[q]\right|^{2}}{\sigma_{n}^{2}+\sum_{j=1, j \neq i}^{N_{s}}\left|\beta_{i, j}[q]\right|^{2}} .
$$

Clearly, on those subcarriers where $\mathrm{SNR}_{i}[q] \gg$ $\operatorname{SINR}_{i}[q]$, there is a strong indication of significant interfering terms (either because of poor channel conditioning and/or mismatched transmit and receive filters due to imperfect CSIT) that would favour the application of MLD. With this observation in mind, Algorithm 1 can be used to decide which detection scheme should be selected on the $q$ th subcarrier. To this end, the algorithm evaluates expressions (10) and (11) on each subcarrier for each 
spatial mode and, utilising the mapping $\mathcal{F}: \Re^{N_{s}} \rightarrow \Re$, derives two metrics, one for the overall SNR and another one for the overall SINR, on which the detection decision for that subcarrier will be based. Potential mapping functions to be used are the mean, minimum or maximum of the incoming $N_{s}$-long vector. Parameter $\alpha \in[0,1]$ in Algorithm 1 is used to allow a certain amount of interference to be present yet still relying on MMSE detection. It effectively acts as a complexity-performance trading knob: as $\alpha \rightarrow 0$, the more interference is allowed and the more often the system relies on MMSE detection (lower complexity and poorer performance than with ML). Two important remarks are in place:

- The subcarrier-based nature of the algorithm is to be emphasised. Most likely, for a given packet, some of the subcarriers will be linearly detected while others will require the use of MLD.

- Sphere detection-based MLD usually starts the search procedure using the zero forcing (ZF) solution as the centre of the sphere. However, if an estimate of the noise power $\sigma^{2}$ is available, centering the search around the MMSE solution is computationally advantageous [20]; thus, the computation of (6), even in the case of eventually relying on MLD, still plays a role.

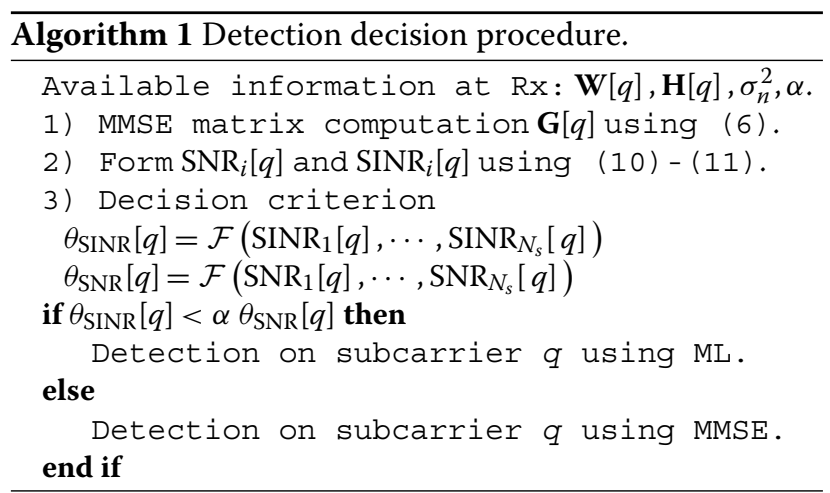

\section{Iterative soft detection}

Despite the importance of hard decoding in its own right, most practical deployments are based on the use of softbased decoding principles. Consequently, it is important to consider the performance of the proposed adaptive detection scheme when the component detectors extract soft information, typically in the form of log-likelihood ratios (LLRs), from the received samples. Furthermore, soft detectors are often able to operate iteratively following turbo receiver design principles. In this case, the MIMO detector and channel decoder exchange (soft) information back and forth with the corresponding LLRs becoming more reliable at each iteration [21]. The next subsections describe two popular soft-based detection schemes, one based on MLD and another one based on MMSE, and the iterative extension within the context of the considered setup.

\subsection{MLD-based soft detection}

For the case of ML detection based on the sphere decoder, the authors in [21] introduced the list sphere decoder (LSD) that not only renders the most likely (hard) estimate $\tilde{\mathbf{s}}[q]^{\mathrm{ML}}$ but also provides a list of the closest candidate points to the ML solution. This list enables the derivation of soft information in the form of LLR for each bit. To this end, the transformation $\mathbf{s}[q]=\mathcal{M}(\mathbf{b})$ is defined as the modulation mapping to arrive at symbol vector $\mathbf{s}_{i}[q]$ from the corresponding $\operatorname{bits}^{\mathrm{c}} \mathbf{b}=\left(b_{1} b_{2} \ldots b_{N_{b}}\right)^{T}$ where $N_{b}=\log _{2} M$. Making use of the max-log approximation, the LLR for a given bit $b_{p}$ (belonging to an arbitrary subcarrier $q$ and stream $i$ ) can be approximated by [21]

$$
\begin{aligned}
\mathcal{L}_{i}^{\mathrm{ML}}[q]\left(s_{i}[q], b_{p}\right) & \\
\approx & \frac{1}{2} \max _{\mathbf{b} \in \mathcal{B}_{p,+1}}\left\{-\frac{1}{\sigma_{n}^{2}}\|\mathbf{r}[q]-\mathbf{A}[q] \mathcal{M}(\mathbf{b})\|^{2}\right\} \\
& -\frac{1}{2} \max _{\mathbf{b} \in \mathcal{B}_{p,-1}}\left\{-\frac{1}{\sigma_{n}^{2}}\|\mathbf{r}[q]-\mathbf{A}[q] \mathcal{M}(\mathbf{b})\|^{2}\right\},
\end{aligned}
$$

where the characters $\mathcal{B}_{p,+1}$ and $\mathcal{B}_{p,-1}$ represent the sets of $2^{N_{b}-1}$ bit vectors whose $p^{\text {th }}$ position is a ' +1 ' or ' -1 ', respectively. Moderate values of $M$ and/or $N_{s}$ make the sets $\mathcal{B}_{p,+1}$ and $\mathcal{B}_{p,-1}$ extremely large, making the search in (12) computationally challenging. To address this issue, the LSD limits the search to the sets $\hat{\mathcal{B}}_{p,+1}=\mathcal{B}_{p,+1} \cap \mathcal{C}$ and $\hat{\mathcal{B}}_{p,-1}=\mathcal{B}_{p,-1} \cap \mathcal{C}$ where $\mathcal{C}$ is the set containing the bit vectors corresponding to the $N_{\text {cand }}$ candidates closer, in a Euclidean sense, to the received samples, i.e. $\mathcal{C}=\left\{\mathbf{b}^{1}, \ldots, \mathbf{b}^{N_{\text {cand }}}\right\}$ where $\mathbf{b}^{n}=\mathcal{M}^{-1}\left(\tilde{\mathbf{s}}^{[c]}[q]\right)$ with $\left\{\tilde{\mathbf{s}}^{[1]}[q], \ldots, \tilde{\mathbf{s}}^{\left[N_{\text {cand }}\right]}[q]\right\}$ being the $N_{\text {cand }}$ group candidates for which the Euclidean distance $\|\mathbf{r}[q]-\mathbf{A}[q] \mathbf{s}[q]\|^{2}$ is smallest.

\subsection{MMSE-based soft detection}

In order to derive soft estimates from the MMSE-detected samples, the procedure described in [22] is adapted to the situation at hand. To this end, let us define the post-MMSE receive filter SNR for an arbitrary subcarrier $q$ and stream $i$ as

$$
\operatorname{SNR}_{i}[q]^{\mathrm{MMSE}}=\frac{1}{\left[\left(\frac{1}{\sigma^{2}} \mathbf{A}[q] \mathbf{A}^{H}[q]+\mathbf{I}_{N_{R}}\right)^{-1}\right]_{i, i}}-1 .
$$


Based on [22], the LLR for the in-phase bit on the $p$ th position of the symbol stream $i$ is given by

$$
\mathcal{L}_{i}^{\mathrm{MMSE}}[q]\left(s_{i}[q], b_{I, p}\right)=\frac{\mathrm{SNR}_{i}[q]^{\mathrm{MMSE}}}{4} D_{I, p},
$$

where $D_{I, p}$ is given by the mappings defined in [22] (14 to 18). LLRs for the bits in quadrature are computed using an analogous procedure.

Note that the extraction of the soft information is much more involved in the case of MLD-based processing, even when employing the lower-complexity LSD, than for the MMSE-based detector. As shown in Figure 3 and ignoring for now the iterative processing (shaded region), the resulting LLR streams $\left\{\mathcal{L}_{1}^{D 1}, \ldots, \mathcal{L}_{N_{s}}^{D 1}\right\}$, whose entries are given by $\mathcal{L}_{i}^{\mathrm{ML}}$ or $\mathcal{L}_{i}^{\mathrm{MMSE}}$ depending on the detection procedure employed for the corresponding subcarrier, are subsequently de-segmented, de-interleaved, de-parsed and de-punctured to form the coded LLR stream that is finally supplied to a Viterbi decoder to yield the estimated information bits.

\subsection{Channel-aware iterative soft detection}

Further performance improvements in the form of lower error rates can be achieved if the detector and channel decoder are allowed to exchange information, specially when the detector is based on ML detection principles [21]. In fact, it has been observed that when detection relies on linear processing techniques such as MMSE, the benefits of iterative reception become rather marginal [23]. Consequently, in this work, the application of iterative processing is limited to those cases where MLD has been selected as the preferred detection strategy.

As shown in Figure 3, each (subcarrier-based) detector operates in accordance with Algorithm 1 to decide which detection strategy, MLD or MMSE, should be used and computes the corresponding LLR for each bit using (12) or (14), respectively. The de-segmentation process is then in charge of collecting the LLR values computed for the successive OFDM symbols forming a packet/stream resulting in the LLR streams $\left\{\mathcal{L}_{1}^{D 1}, \ldots, \mathcal{L}_{N_{s}}^{D 1}\right\}$. These LLRs, after subtracting any a priori knowledge available from previous iterations, give rise to the extrinsic information $\left\{\mathcal{L}_{1}^{E 1}, \ldots, \mathcal{L}_{N_{s}}^{E 1}\right\}$, which after suitable de-interleaving and spatial de-parsing, results in the input stream to the maximum a posteriori (MAP) decoder $\left(\mathcal{L}_{1}^{A 2}\right)$. The MAP decoder has a double output: on one hand, an estimate of the information symbols, and on the other hand, a refined version of the input LLRs. This latter output, $\mathcal{L}_{1}^{D 2}$, after subtracting already known information $\left(\mathcal{L}_{1}^{A 2}\right)$, results in the extrinsic information $\mathcal{L}_{1}^{E 2}$ to be fed back to the detection stage. To this end, signal $\mathcal{L}_{1}^{E 2}$ is suitably parsed and interleaved resulting in the sequences $\left\{\mathcal{L}_{1}^{A 1}, \ldots, \mathcal{L}_{N_{s}}^{A 1}\right\}$ forming the a priori information for the next turbo iteration. Note that only the LLRs corresponding to those subcarriers that have been detected using MLD are fed back to the detector (denoted in Figure 3 by $\left\{\mathcal{L}_{i}^{A 1}\right\}_{\mathrm{ML}}$ ) while no information is fed back to the MMSE-detected subcarriers.

\section{Numerical results}

\subsection{Simulation setup}

The simulation environment has been defined in accordance with specifications from the IEEE 802.11n architectures [18], considering a setup with $N_{T}=N_{R}=4$ antennas transmitting $N_{s}=4$ streams. The system operates on a bandwidth of $B=20 \mathrm{MHz}$ using $N_{c}=64$ subcarriers out of which $N_{d}=52$ are used for data transmission and the rest are devoted to pilot signalling and guard bands. For all simulations, transmission modes

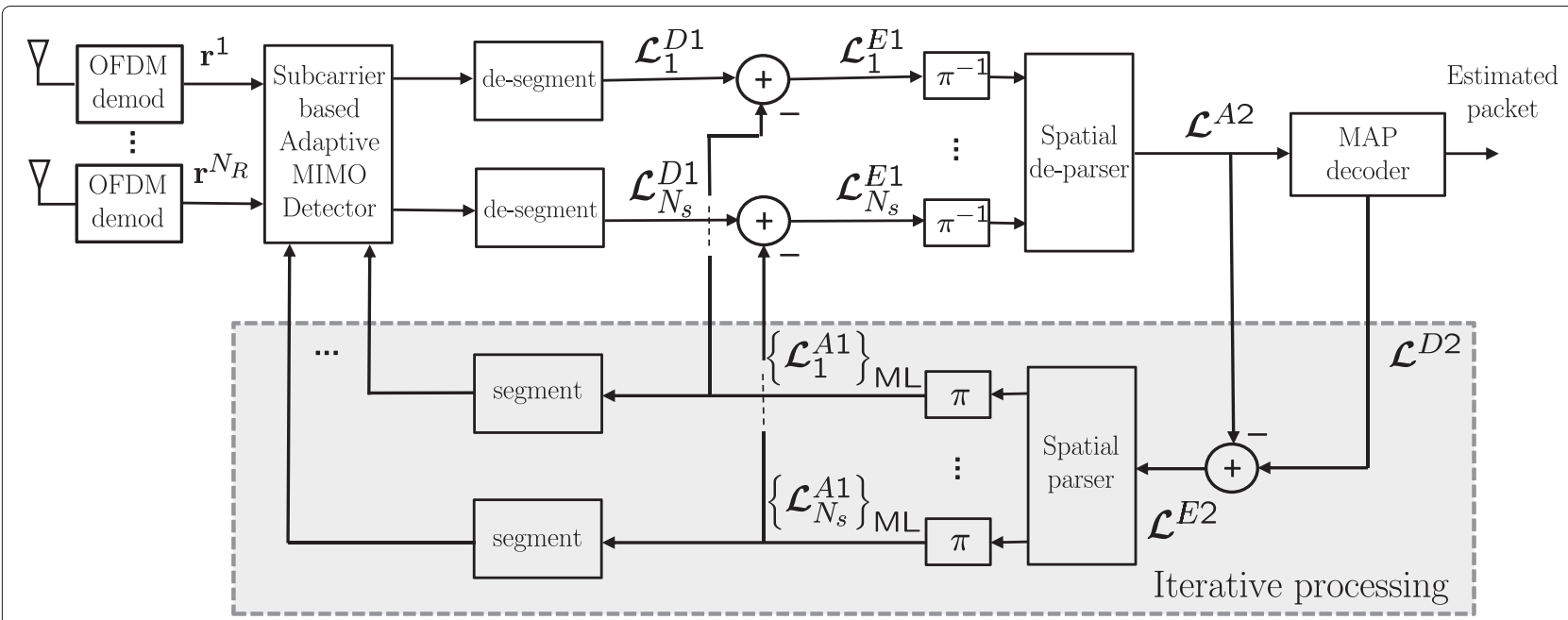

Figure 3 Channel-aware turbo receiver. 
with either quadrature phase shift keying (QPSK) or 16QAM modulation and a 1/2-rate convolutional channel coder with generator polynomials $\boldsymbol{g}=[133,171]_{8}$ have been employed. Full cyclic prefix is used in order to guarantee the avoidance of interference among successively transmitted OFDM symbols. Power allocation matrices are computed according to the ARITH-MSE criterion in [7] for hard decoding and uniform power allocation for soft/iterative decoding ${ }^{\mathrm{d}}$. Two different spatial spreading matrices have been considered, $\mathbf{C}=\boldsymbol{I}_{N_{s}}$ (no spreading) and $\mathbf{C}=\boldsymbol{\Psi}_{N_{s}}$ (full spreading), with $\boldsymbol{I}_{N_{s}}$ and $\boldsymbol{\Psi}_{N_{s}}$ denoting the identity and rotated Walsh-Hadamard matrices of dimension $N_{s}$, respectively.

Interestingly, IEEE 802.11-based systems are a representative scenario where the CSIT accuracy may (widely) vary over a short time frame. This is due to the channel contention mechanism that, based on CSMA/CA, causes the time span between the reception of channel-related feedback at $\mathrm{Tx}$ and its utilisation to fluctuate on a packet basis and, moreover, to make it heavily dependent on the number of active users in the system. Note that when users enter or exit the system, the average delay in using the acquired CSIT for the rest of the active users is likely to vary, thus effectively implying a degradation or improvement in the CSIT accuracy. For the results shown here, the channel, generated following the specifications in [24], is assumed to remain static over the duration of a packet and vary independently from packet to packet (block fading).

Without loss of generality, it is assumed in (3) that $\rho=1-\varphi$ where $\varphi$ is a random variable with a gamma probability density function $\Gamma(\mu, 1)$ truncated to the interval $[0,1]$. In particular, the results here are presented for $\mu=0.015, \mu=0.37$ and $\mu=1.31$, which lead to average values of $\bar{\rho}=0.99, \bar{\rho}=0.8$ and $\bar{\rho}=0.5$, respectively. Figure 4 depicts histograms for 2,500 realisations (i.e. packets) of the resulting $\rho$ parameter for the three different values of $\mu$. It can clearly be appreciated that, indeed, a value of $\mu=0.015$ results in a very accurate CSIT for most of the frames, whereas when $\mu=0.37$ or $\mu=1.31$, the corresponding $\rho$ values clearly suggest that a significant proportion of packets are transmitted with a rather imperfect CSIT. Note that the generation of $\rho$ based on the gamma distribution allows the modelling of various network operating conditions just by adjusting a single parameter $(\mu)$. Algorithm 1 parameters for the hard-decoded setup have been chosen to be $\alpha \in\{0.25,0.5,0.75\}$ while $\mathcal{F}(\mathbf{x})=\min (\mathbf{x})$, and for the softdecoded setup, $\alpha \in\{0.75,0.85,0.95\}$ and $\mathcal{F}(\mathbf{x})=\min (\mathbf{x})$. Lastly, for the iterative decoding configuration, Algorithm 1 is configured with $\mathcal{F}(\mathbf{x})=\min (\mathbf{x})$ and $\alpha=0.975$. The different choice of $\alpha$ for the hard, soft and iterative decoding is due to the very different SNR levels each of these configurations operates in that affects what can be regarded as a tolerable level of interference or not. The use of other mapping functions such as $\mathcal{F}(\mathbf{x})=\max (\mathbf{x})$ or $\mathcal{F}(\mathbf{x})=\operatorname{mean}(\mathbf{x})$ has also been tested, but the corresponding results do not differ substantially from the ones presented next using $\mathcal{F}(\mathbf{x})=\min (\mathbf{x})$.

\subsection{Hard-decoded results}

Figure 5 presents results for the non-spread setup. The left plot shows the PER performance when very accurate CSIT is available, and as expected under these conditions, the linear receiver in (6) virtually diagonalises the overall processing chain, thus making linear detection nearly optimal. Only for those very few cases where CSIT error is significant, MLD provides an advantage over MMSE, a situation leading to the rather small gain observed between the performance of both fixed detectors. The performance for the adaptive detector following Algorithm 1 remains
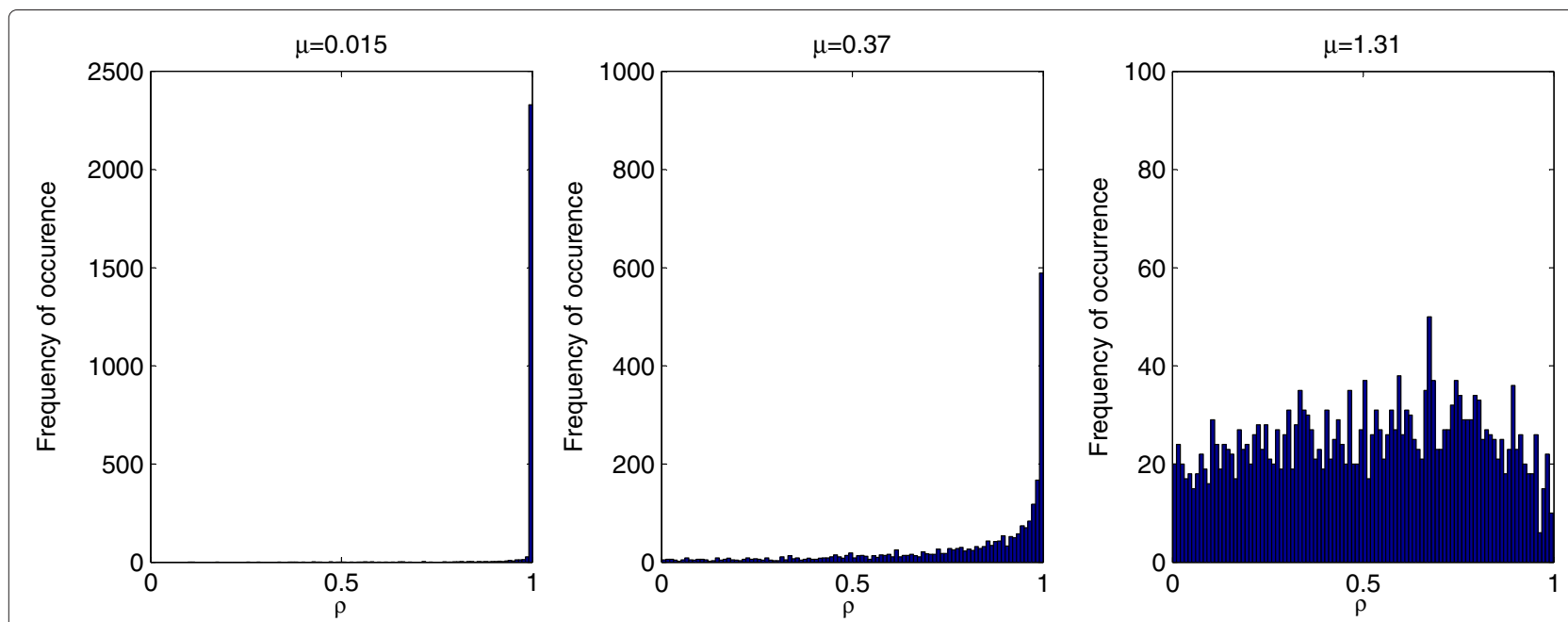

Figure 4 Histogram of $\rho$ derived from 2,500 independent draws when $\mu=0.015$ (left), $\mu=0.37$ (centre) and $\mu=1.31$ (right). 

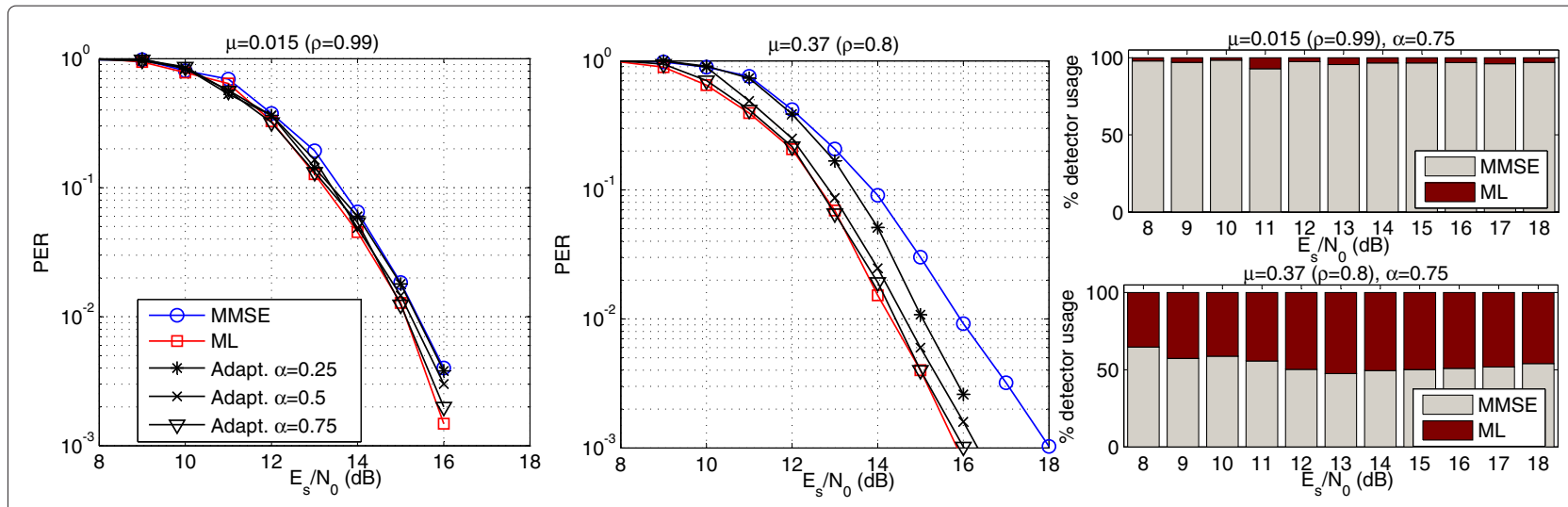

Figure 5 PER and complexity for setup without spatial spreading $\left(C=I_{N_{s}}\right)$ using hard decoding. QPSK modulation.

unaltered regardless of the value of $\alpha$. The top-right plot in the same figure shows the percentage of utilisation of each detector for the particular case of $\alpha=0.75$, and remarkably, Algorithm 1 overwhelmingly chooses the linear approach for any SNR level. When CSIT quality diminishes, differences among the PER performance of the various detectors appear, as shown in the central plot in Figure 5. There are two noticeable facts regarding the performance of MLD: firstly, it now clearly outperforms linear detection, and secondly, it improves upon the results obtained using very accurate CSIT. This somewhat counterintuitive effect, already observed and discussed in [15], is caused by the absence of spatial spreading: the mismatch between $\mathrm{Tx}$ and $\mathrm{Rx}$ due to a lousy CSIT causes significant interfering terms to appear in (9) that effectively act as a form of spreading that the MLD can exploit, resulting in a $2-\mathrm{dB}$ gain over linear processing. The adaptive detector, depending on the value of $\alpha$, exhibits various degrees of performance. For the particular choice of $\alpha=0.75$, it basically achieves optimum performance while, as shown on the bottom-right plot of Figure 5, it only triggers the use of MLD for about $40 \%$ to $50 \%$ of the detector invocations with the rest of the times relying on linear processing.

Results in Figure 6 correspond to a setup employing rotated Walsh-Hadamard spatial spreading. In this case, either with accurate or inaccurate CSIT, MLD clearly outperforms linear detection by up to $4 \mathrm{~dB}$ (left and central plots in Figure 6). The adaptive detector is seen to yield different error rates depending on the chosen $\alpha$. For the particular choice of $\alpha=0.75$, the attained PER is within $0.5 \mathrm{~dB}$ of the optimal solution (fixed MLD) while requiring only the use of MLD for about $40 \%$ of the detector invocations when CSIT is accurate and for around $60 \%$ when this is rather inaccurate.

\subsection{Soft-decoded results}

For the soft-decoded case, only non-spread configurations are considered $\left(\mathbf{C}=\mathbf{I}_{N_{s}}\right)$ as it has been shown in [14,25] that, in soft-based BICM systems, spatial spreading leads
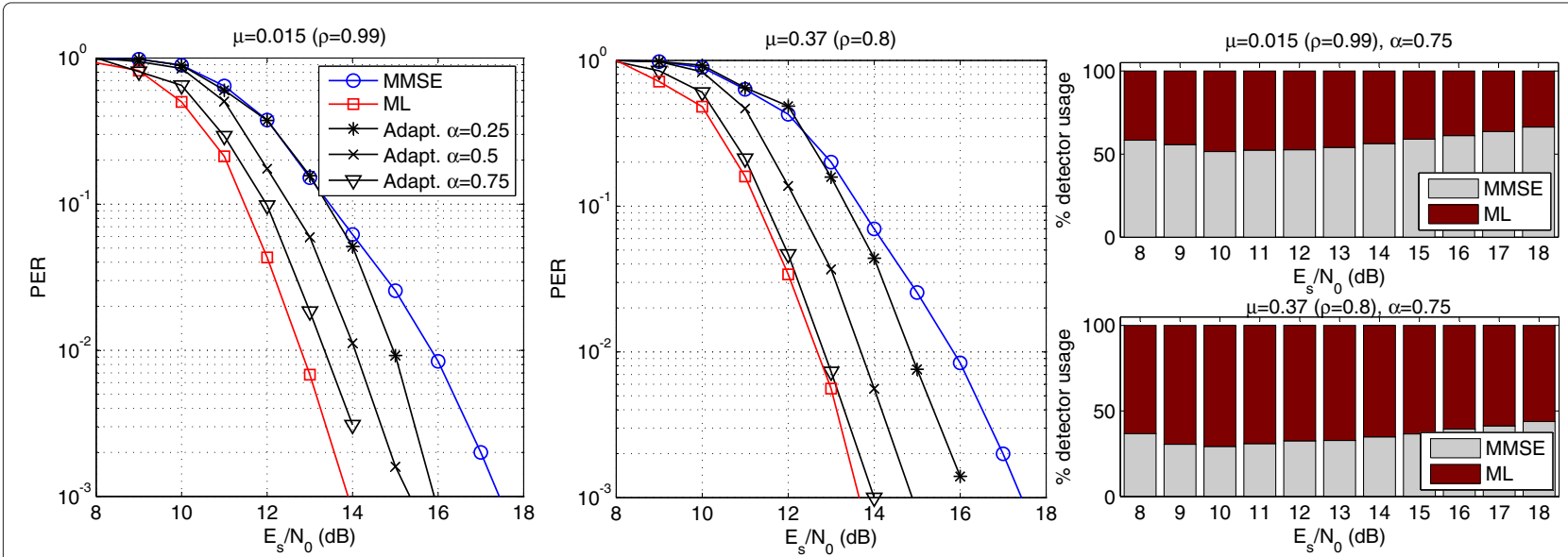

Figure 6 PER and complexity for setup with rotated Walsh-Hadamard spatial spreading $\left(C=\Psi_{N_{s}}\right)$ using hard decoding. QPSK modulation. 
to a PER performance degradation. PER performance and complexity results are shown in Figure 7 for the three considered levels of CSIT accuracy when employing softbased decoding. Focusing on the PER performance, it can be observed that the more inaccurate the CSIT is, the bigger the difference between MLD and MMSE detection becomes. In particular, all detectors achieve similar performance for $\mu=0.015$ (top-left plot in Figure 7) whereas for $\mu=0.37$, and even more markedly when $\mu=1.31$, the performance gap between ML and MMSE is somewhere between 2 and $3 \mathrm{~dB}$ for the typically relevant range of PER values $\left(10^{-3}\right.$ to $\left.10^{-1}\right)$. The adaptive detector behaves as expected: for near-perfect CSIT, similar PER performance was obtained independent of $\alpha$ $(\alpha \in\{0.75,0.85,0.95\})$, while under imperfect CSIT, the performance (and complexity) of the adaptive detector is greatly influenced by the particular choice of $\alpha$. The bottom plots in Figure 7 represent the percentages of detector utilisation when employing Algorithm 1 with $\alpha=0.95$ for the three values of $\mu$. Notice that large values of $\alpha$ such as this one strive for performance optimisation. As anticipated, when CSIT is nearly perfect, the adaptive detector relies almost exclusively on MMSE detection. In contrast, as CSIT accuracy degrades, the adaptive detector tends to favour the more frequent use of MLD to the point that for very poor-quality CSIT information, the adaptive detector almost invariably triggers the use of MLD. Nevertheless, note that, although not shown here and as in the hard-decoded scenario, a lower value of $\alpha$ would lead to significant computational savings (more frequent use of MMSE for $\mu=0.37$ or $\mu=1.31$ ) at the cost of a slight degradation in PER performance.

\subsection{Iteratively decoded results}

Figure 8 shows results when iterative decoding is applied. For this scenario, the system was configured to use 16-QAM modulation, no spatial spreading was in use $\left(\mathbf{C}=\mathbf{I}_{N_{s}}\right)$ and the adaptive detector's threshold was set to $\alpha=0.975$. Results are shown for two different degrees of statistical CSIT quality, $\mu=0.015$ and $\mu=1.31$, corresponding to very accurate and rather inaccurate channel knowledge, respectively. The first remarkable point to note is that, as noted in previous results, when the transmitter can rely on a good channel estimate $(\mu=$ 0.015), there are no very large differences between the (non-adaptive) ML and MMSE soft detectors, with only a marginal benefit for the ML-based receiver as shown in the left plot in Figure 8. When iterative detection is allowed $^{\mathrm{e}}$, the ML detector is able to somewhat improve performance over its non-iterative counterpart. Nonetheless, it is remarkable that the SNR gap between the low-complexity (non-iterative) MMSE-based soft detector and the much more complex iterative ML receiver is less than $1 \mathrm{~dB}$. When the detection strategy on each subcarrier is selected according to Algorithm 1, it can be clearly observed that for the vast majority of cases, MMSE detection is selected (see top-right plot in Figure 8) with ML resulting in the chosen strategy only for those few cases where there was a considerable mismatch between the CSIT and the true channel. Consequently, the performance of the adaptive detector lies in the narrow ground between that of the fixed receivers. As shown in the figure, the adaptive detector was tested with and without the possibility of iterating, and as with the fixed ML decoder, it was observed that iterating resulted in a rather marginal
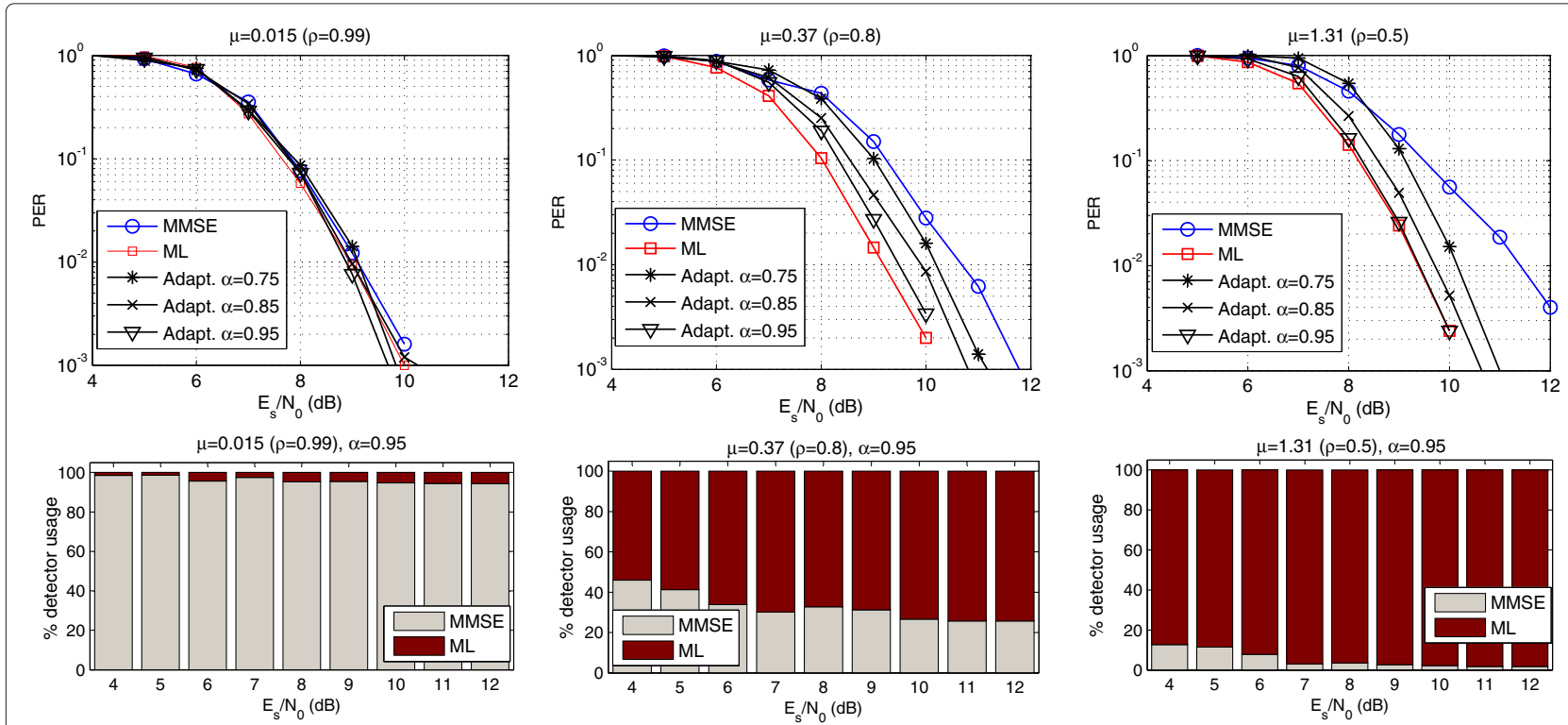

Figure 7 PER and complexity for setup with no spatial spreading $\left(C=I_{N_{s}}\right)$ using soft decoding. QPSK modulation. 


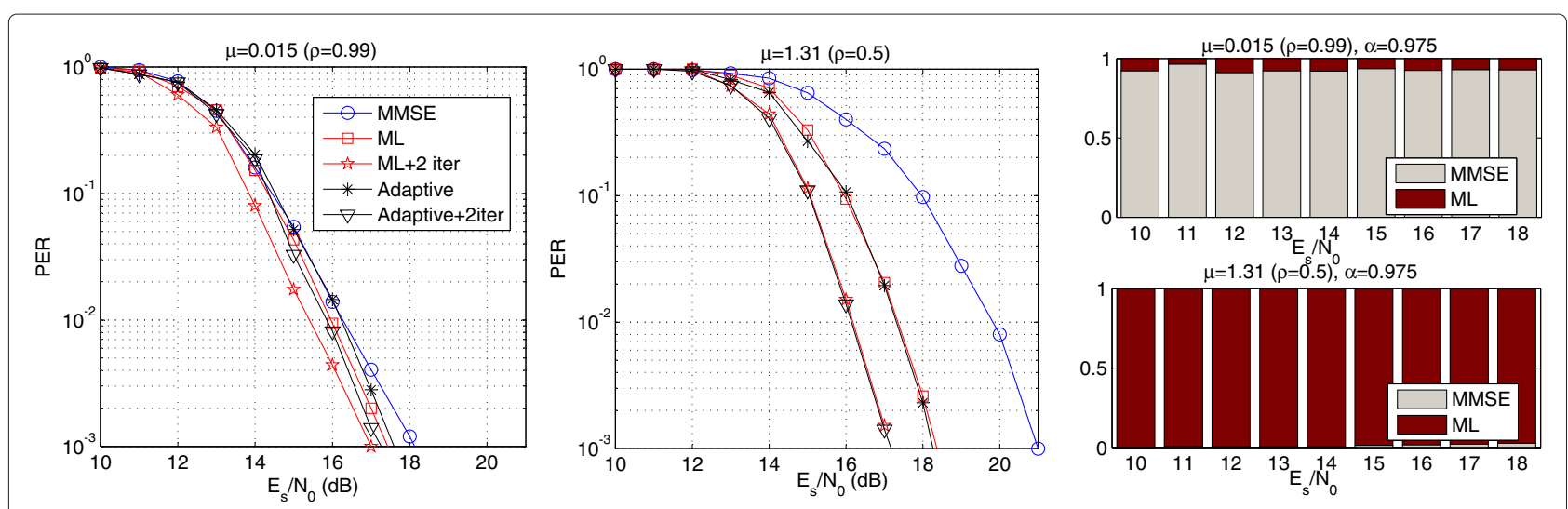

Figure 8 PER and complexity for setup with no spatial spreading $\left(C=I_{N_{s}}\right)$ using iterative decoding. 16-QAM modulation.

improvement. Note that as mentioned in Section 4.3, when using the channel-aware iterative detector, information feedback is only conducted for those subcarriers employing ML detection.

When the statistical CSIT quality is considerably degraded $(\mu=1.31)$, results are significantly different. As it can be observed in the middle plot of Figure 8, and in line with results in the previous subsection, noniterative ML detection provides a gain of nearly $3 \mathrm{~dB}$ with respect to MMSE. Furthermore, when the ML receiver is allowed to iterate, a further 1 - to $1.5-\mathrm{dB}$ gain is achieved. When using channel-aware detection, it is observed that Algorithm 1, either with or without iterations, rightly chooses to rely on ML detection owing to the rather large interfering terms the receiver observes when evaluating (10) and (11).

\subsection{An important remark}

It is worth emphasising that the merits of the adaptive detector should be valued by globally appreciating the results on each of the considered scenarios (hard, soft and iterative decoding): a single configuration for each type of receiver $(\alpha=0.75, \mathcal{F}(\mathbf{x})=\min (\mathbf{x})$ for hard decoding, $\alpha=0.95, \mathcal{F}(\mathbf{x})=\min (\mathbf{x})$ for soft decoding and $\alpha=0.975, \mathcal{F}(\mathbf{x})=\min (\mathbf{x})$ for iterative decoding $)$ leads to a strategy able to attain virtually optimum PER performance while potentially offering a very significant complexity reduction with respect to the full use of MLD. In other words, Algorithm 1 provides the receiver with the capability of distinguishing when MLD will be effective and when MMSE will suffice. This scheme can therefore be very attractive in those scenarios where the quality of CSIT may vary over time such as it occurs in today's WLAN environments depending on the number of users in the system or changes in the environment. Furthermore, note that the parameter $\alpha$ acts as a performance/complexity trading knob, thus enabling the reconfiguration of the system as a function of, for instance, the available battery power or required processing latency.

\section{Conclusions}

This paper has proposed an adaptive detection technique that allows the receiver of a MIMO-OFDM linearly precoded system to toggle between the use of MMSE and MLD depending on the CSIT accuracy and/or channel conditioning. The introduced technique works on a persubcarrier basis and is compatible with different receiver architectures, namely hard, soft and iterative decoding schemes. Numerical results have shown that regardless of the receiver setup, the adaptive detector is able to distinguish the system conditions that allow MLD to boost performance from those where the much simpler MMSE detector would perform (near) optimally.

\section{Endnotes}

${ }^{a}$ The incorporation of antenna correlation effects to the current system model is trivial; however, it unnecessarily complicates notation without providing any further insight or significantly altering numerical results.

bThis is a realistic assumption since the receiver should be aware of the last CSI information sent to the transmitter. Alternatively, if the transmitter sends pilot symbols through the precoder (and channel), the receiver can also deduct the precoding filter used in transmission.

'To simplify the notation, the subcarrier and stream indices are skipped when referring to the bits.

${ }^{\mathrm{d}}$ The (possibly iterative) utilisation of soft information at the receiver suggests using capacity-based measures to optimise the power allocation. Unfortunately, under imperfect CSIT, no closed-form solutions are available and power allocation solutions require convex numerical optimisation procedures (see [5] for a detailed discussion on this issue). 
${ }^{\text {e}}$ For the results shown here, only two iterations between detector and decoder were allowed as it was observed that further iterations did not bring along any significant performance benefit.

\section{Competing interests}

The authors declare that they have no competing interests.

\section{Acknowledgements}

Work funded by MINECO and FEDER under project AM3DIO (TEC2011-25446), Spain.

Received: 14 May 2013 Accepted: 24 September 2013

Published: 7 October 2013

\section{References}

1. G Stuber, J Barry, S McLaughlin, Y Li, M Ingram, T Pratt, Broadband MIMO-OFDM wireless communications. Proc. IEEE 92, 271-294 (2004)

2. A Paulraj, D Gore, R Nabar, H Bolcskei, An overview of MIMO communications-a key to gigabit wireless. Proc. IEEE 92, 198-218 (2004)

3. A Ghosh, R Ratasuk, B Mondal, N Mangalvedhe, T Thomas, LTE-advanced: next-generation wireless broadband technology. Wireless Commun. IEEE 17(3), 10-22 (2010)

4. D Gesbert, M Kountouris, R Heath, C-B Chae, T Salzer, Shifting the MIMO paradigm. IEEE Signal Process. Mag. 24(5), 36-46 (2007)

5. M Vu, A Paulraj, MIMO wireless linear precoding. IEEE Signal Process. Mag. 24(5) (2007)

6. M Costa, Writing on dirty paper. IEEE Trans. Inf. Theory 29(3), 439-441 (1983)

7. D Palomar, J Cioffi, M Lagunas, Joint Tx-Rx beamforming design for multicarrier MIMO channels: a unified framework for convex optimization. IEEE Trans. Signal Process. 51(9), 2381-2401 (2003)

8. E Sengul, E Akay, E Ayanoglu, Diversity analysis of single and multiple beamforming. IEEE Trans. Commun. IEEE Trans. 54(6), 990-993 (2006)

9. L Ordoñez, D Palomar, A Pages-Zamora, J Rodriguez Fonollosa, High-SNR analytical performance of spatial multiplexing MIMO systems with CSI. IEEE Trans. Signal Process. 55(11), 5447-5463 (2007)

10. HJ Park, B Li, E Ayanoglu, Constellation precoded multiple beamforming IEEE Trans. Commun. 59(5), 1275-1286 (2011)

11. X Zhang, D Palomar, B Ottersten, Statistically robust design of linear MIMO transceivers. IEEE Trans. Signal Process. 56(8), 3678-3689 (2008)

12. E Sengul, H Park, E Ayanoglu, Bit-interleaved coded multiple beamforming with imperfect CSIT. IEEE Trans. Commun. 57(5), 1505-1513 (2009)

13. X Yuan, C Xu, L Ping, X Lin, Precoder design for multiuser MIMO ISI channels based on iterative LMMSE detection. IEEE J. Selected Top. Signal Process. 3(6), 1118-1128 (2009)

14. F Riera-Palou, G Femenias, in Proceedings of IEEE SPAWC 2011. On detection strategies for linearly precoded MIMO-OFDM systems (San Francisco, 26-29 June 2011)

15. F Riera-Palou, G Femenias, in Proceedings of IEEE CISS 2012. On optimal detection for linearly precoded MIMO-OFDM systems with imperfect CSIT (Princeton, 21-23 March 2012)

16. U Fincke, M Pohst, Improved methods for calculating vectors of short length in a lattice, including a complexity analysis. Math. Comput. 44, 463-471 (1985)

17. B Hassibi, H Vikalo, On the sphere-decoding algorithm I. Expected complexity. IEEE Trans. Signal Process. 53(8), 2806-2818 (2005)

18. IEEE Standards Board, IEEE draft standard for information technology-telecommunications and information exchange between systems-local and metropolitan area networks-specific equirements-part 11: wireless LAN medium access control (MAC) and physical layer (PHY) specifications amendment: enhancements for higher throughput. IEEE Approved Draft Std P802.11n/D11.0 (IEEE, Piscataway, 2009)

19. M Matthaiou, D Laurenson, C-X Wang, in International Proceedings of Wireless Communications and Mobile Computing Conference, 2008. IWCMC '08. Reduced complexity detection for Ricean MIMO channels based on condition number thresholding (Crete, 6-8 August 2008)

20. Q Liu, L Yang, A novel method for initial radius selection of sphere decoding. IEEE Vehicular Technology Conference. 2, 1280-1283 (2004)
21. B Hochwald, S ten Brink, Achieving near-capacity on a multiple-antenna channel. IEEE Trans. Commun. 51(3), 389-399 (2003)

22. F Tosato, P Bisaglia, in Proceedings of the IEEE ICC. Simplified soft-output demapper for binary interleaved COFDM with application to HIPERLAN/2 (New York, 2002)

23. E Zimmermann, G Fettweis, in Proceedings of IEEE PIMRC. Adaptive vs. hybrid iterative MIMO receivers based on MMSE linear and soft-SIC detection (Helsinki, 11-14 September 2006)

24. V Erceg, WLAN TGn Channel Models. IEEE 802.11-03/940r4, Draft proposal, May 2004

25. Y Kim, JM Cioffi, K Holt, in IEEE Wireless Telecommunications Symposium, 2006. WTS '06. Low complexity BICM-OFDM-based MIMO receiver using successive interference cancelation (Los Angeles, 2006)

doi:10.1186/1687-1499-2013-240

Cite this article as: Riera-Palou and Femenias: Channel-aware adaptive receivers for linearly precoded MIMO-OFDM systems with imperfect CSIT. EURASIP Journal on Wireless Communications and Networking 2013 2013:240.

\section{Submit your manuscript to a SpringerOpen ${ }^{\mathcal{O}}$ journal and benefit from:}

- Convenient online submission

Rigorous peer review

- Immediate publication on acceptance

- Open access: articles freely available online

- High visibility within the field

- Retaining the copyright to your article

Submit your next manuscript at $>$ springeropen.com 